\title{
Addressing photoacoustics standards
}

To the Editor - Photoacoustic imaging (PAI) is a rapidly emerging imaging modality that enables optical absorption contrast to be visualized at high spatial resolution across length scales ranging from single organelles to intact living animals. Over the last two decades, PAI has been widely applied in preclinical studies, where it has been used to map the distribution of endogenous chromophores, such as haemoglobin, melanin and lipids, in living animals. Building on these successes, PAI has now entered the clinical realm and a wealth of scientific publications has demonstrated its potential in aiding studies of cancer, Crohn's disease and arthritis, as well as other medical conditions. While several commercial clinical instruments are now available, there is an urgent need for standardized methodology that enables accurate and reproducible comparisons of the performance of different instruments. This is required for quality assurance and control, as well as for technical validation of the precision and accuracy of PAI biomarkers.

We wish to inform the readers of Nature Photonics that we have founded the International Photoacoustic Standardisation Consortium (IPASC) to address this need. IPASC was born out of community-wide discussions during the past two Photons Plus Ultrasound meetings at the SPIE Photonics West conference. The overall goal of IPASC is to reach an international consensus on PAI standardization to improve the quality of preclinical studies and to accelerate efforts in clinical translation. IPASC aims to: (1) define widely accepted test objects ('phantoms') for use with preclinical and clinical PAI systems; (2) provide open, publicly available, reference datasets for testing of data reconstruction and spectral processing algorithms in a widely accepted data format; (3) use these phantoms to enable quantitative comparison of PAI data acquired with different system designs and analysed with different data reconstruction and spectral processing algorithms; and (4) agree upon a standardized and validated test method for new PAI instruments to help with comparison of published results.

IPASC is currently represented globally in 14 countries with 52 academic and 16 industrial members. Our academic members have scientific interests ranging from instrumentation development to clinical application, while our industrial members include commercial vendors of PAI instruments as well as laser manufacturers, among others. Over the past year, IPASC convened a series of working groups, which met regularly to identify key challenges for PAI standardization and important directions for future work. The resulting findings and documents were recently presented at the Photons Plus Ultrasound meeting at SPIE Photonics West on 5 February 2019 and are available on our website at https://www.ipasc.science. Based on the following panel discussion, IPASC efforts have been streamlined into three thematic areas: phantom development; data acquisition and management; and study design (Fig. 1).

The phantom development theme is tasked with optimizing candidate phantom materials to achieve aim (1). Researchers contributing to this theme are actively developing experimental approaches for phantom fabrication, for which gel wax and polyvinylchloride plastisols were identified as currently the most promising stable phantom materials by the working groups. They are also establishing consensus on methodology to evaluate optical, acoustic, thermal and mechanical properties of PAI phantoms, as well as designing versatile phantom geometries suitable for a range of PAI system configurations.

The data acquisition and management theme focuses on enabling open access and open use of data acquired in IPASC studies to achieve aim (2). Correct post-processing of raw PAI data requires knowledge of the system configuration, including illumination and detection geometries, parameters that have already been captured from consortium members. HDF5 has been identified as a scalable data format suitable for standardized capture of raw time-series data and appropriate metadata. Participants are currently working to develop a unified representation to enable a device-independent approach to data handling and to create a software tool that can convert data from existing commercial PAI instruments into the HDF5 format.

The study design theme is creating methodology and standard operating procedures to achieve aims (3) and (4). An initial pilot multi-centre study has already been conducted using a test-ship-test methodology based on phantoms fabricated in gel wax and optimized for a cylindrical tomographic imaging geometry. These phantoms were fabricated and tested at the

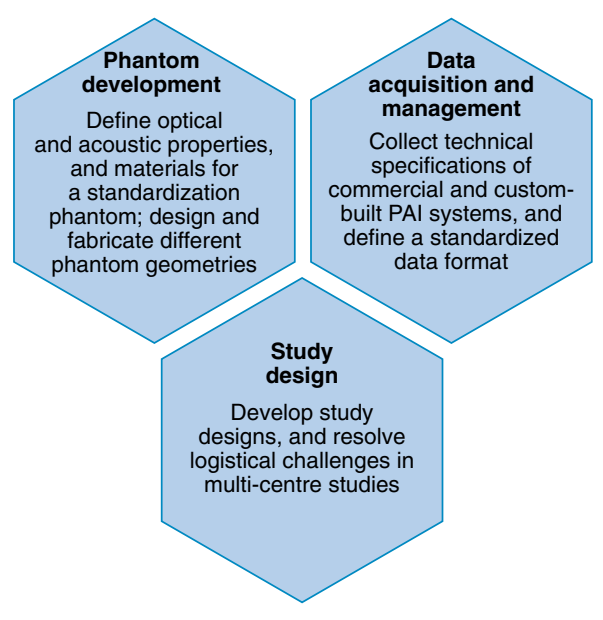

Fig. 1 | Summary of the goals of the IPASC thematic areas.

University of Cambridge and then shipped to eight different sites across the world. The resulting data have helped to direct optimization of the phantom fabrication recipe to improve mechanical and thermal stability and highlighted challenges in data sharing across the consortium, which feed back into the other two themes.

The immediate next steps for IPASC include: (1) using the data from the pilot study to plan and implement the first full multi-centre study, including several PAI system geometries and properties; (2) writing and disseminating an open access publication of the multi-centre study findings and resulting recommendations; (3) creating an open access data repository for PAI researchers; and (4) securing funding to ensure continuity and long-term impact of our efforts.

Most importantly, IPASC is a communityled initiative, so we welcome and value the contributions of new members in developing our thematic activities and achieving our aims. Please visit our website to join the discussion and find out more about our efforts.

\section{Sarah Bohndiek ${ }^{1,2}$ \\ ${ }^{1}$ Department of Physics, University of Cambridge, Cavendish Laboratory, Cambridge, UK. ${ }^{2}$ Cancer Research UK Cambridge Institute, University of Cambridge, Li Ka Shing Centre, Cambridge, UK. e-mail:Seb53@cam.ac.uk}

Written by Sarah Bohndiek on behalf of IPASC.

Published online: 24 April 2019

https://doi.org/10.1038/s41566-019-0417-3 